\title{
Los retos al acceso y uso adecuado de medicamentos
}

\section{Challenges in access to medicine and its proper use}

\author{
Nuria Homedes \\ Professora-associada da School of Public Health/University of Texas. \\ nhomedes@utep.edu
}

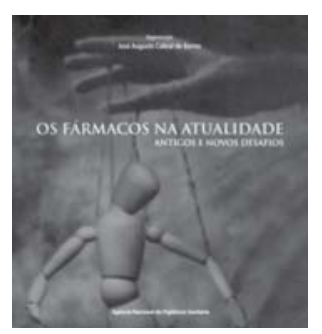

BARROS, José Augusto Cabral de (Comp.). Os fármacos na atualidade: antigos e novos desafios. Brasília: Agência Nacional de Vigilância Sanitária, 2008. 320p. $\int$ osé Augusto Cabral de Barros, profesor del Departamento de Salud Colectiva de la Facultad de Medicina de la Universidad Federal de Juiz de Fora, es uno de los expertos en políticas de medicamentos más reconocidos de América Latina y se ha caracterizado por combinar la reflexión académica con el trabajo comunitario. En 1986, fundó Acción Internacional para la Salud de Latinoamérica y el Caribe (IAS-LAC) y en 1991 la Sociedade Brasileira de Vigilância de Medicamentos (Sobravime). Este libro es una compilación de artículos escritos por expertos europeos y latinoamericanos con el objetivo de proveer ejemplos de iniciativas que han implementado algunas universidades y gobiernos de la región para mejorar el acceso y el uso de los medicamentos, actualizar al lector sobre el contexto global en que se enmarcan las políticas farmacéuticas latinoamericanas y los retos que se deben enfrentar para que la población se beneficie de los medicamentos.

El primer capítulo discute la necesidad de recuperar la perspectiva ética en la definición de políticas de medicamentos. Partiendo de la base de que los medicamentos han tenido una influencia preponderante en los indicadores de salud de la población mundial, se critica el modelo de desarrollo económico, capitalista neoliberal, al que se adscriben las agencias y tratados internacionales y que en el campo de los medicamentos se traduce en políticas que priorizan el lucro de los inversionistas y ejecutivos de la industria farmacéutica al promover el uso exagerado de medicamentos entre los que tienen capacidad de pago y a expensas de privar a muchos del acceso a medicamentos y tecnologías que podrían salvarles la vida.

Tanto en el primer como en el tercer capítulo, escrito por André Martins, se describe la esperanza que desde tiempos inmemoriales el ser humano ha depositado en los remedios milagrosos para restaurar la salud, el bienestar y la belleza; a través de ejemplos se discute cómo esta dependencia ha influido en las estrategias de promoción de medicamentos que utiliza la industria farmacéutica - incluyendo la provisión de información engañosa, la génesis de demanda innecesaria de medicamentos para tratar enfermedades inventadas o exageradas y el uso de medicamentos para mejorar la apariencia física, el desempeño intelectual y deportivo y la interacción social. El doctor Barros llama la atención sobre cómo la industria, para alcanzar sus objetivos, ha conseguido la colaboración de los 
profesionales de la salud, de los gobiernos e incluso de los organismos internacionales, y ha logrado establecer un marco regulatorio global que no incentiva la innovación (sobre todo para enfermedades olvidadas y raras) y dificulta que los gobiernos puedan negociar precios asequibles para los ciudadanos que necesitan medicamentos.

El doctor Martín Cañás ahonda sobre algunos de estos aspectos en el capítulo sexto, donde hace una revisión exhaustiva de la literatura - más de 150 referencias - y demuestra que el arsenal terapéutico existente y la información disponible sobre la eficacia y seguridad de los medicamentos está distorsionada por los intereses de la industria, quién a su vez ha conseguido comprar la complicidad de académicos, científicos y miembros de entidades reguladoras. La lectura de este capítulo cuestiona el uso tan generalizado que se hace del término medicina 'basada en la evidencia', pues parecería que la información disponible es engañosa y que las fuentes confiables de información terapéutica son pocas y no cuentan con los mismos sistemas de divulgación de que goza la industria. El capítulo concluye con ejemplos de las estrategias que están utilizando los países industrializados para remediar la situación.

El libro dedica cuatro capítulos a presentar estrategias para minimizar algunos de los problemas que afectan a la región. En el segundo capítulo, el doctor Barros comenta la iniciativa para promover la investigación de enfermedades olvidadas. En el cuarto, el profesor José María Terán Puente presenta un programa para enseñar a los estudiantes de medicina a analizar y criticar las información de medicamentos que proporciona la industria; y en el quinto, el doctor Fernando Cornejo León describe las diferentes formas que utiliza la industria farmacéutica para captar la lealtad de los futuros prescriptores de medicamentos y enfatiza la importancia de incluir información sobre el uso adecuado de medicamentos y las estrategias de promoción que utiliza la industria en los primeros años de la carrera de medicina. Y en el séptimo capítulo, el doctor Albert Figueras y la doctora Mabel Valsecia, describen cómo los estudios de utilización de medicamentos pueden contribuir a mejorar su uso.

Finalmente, en el último capítulo, la doctora Claudia Vacca discute cómo las políticas de medicamentos genéricos y medicamentos esenciales pueden contribuir a incrementar el acceso a los medicamentos. Este capítulo describe las diferentes definiciones de medicamento genérico y la legislación en torno a los mismos que se encuentra vigente en América Latina. Aclara muchas de las controversias en torno a su calidad y a la aplicabilidad de las pruebas de bioequivalencia.

Si bien la calidad y extensión de los capítulos varía ampliamente, se puede afirmar que se trata de uno de los libros más importantes sobre políticas de medicamentos que se han escrito para la audiencia latinoamericana. Desde mi punto de vista, el capítulo de la doctora Vacca debería ser de lectura obligada para todos los que toman decisiones, académicos, profesionales de la salud y consumidores latinoamericanos, ya que utilizando un estilo muy didáctico aclara conceptos que hasta ahora han impedido o, en el mejor de los casos, frenado la implementación de políticas de medicamentos genéricos en la región. El capítulo del doctor Cañás invita a que los académicos y reguladores latinoamericanos documenten los conflictos de interés que afectan el acceso a la información y al uso adecuado de medicamentos en la región. Solo teniendo información específica se puede convencer a 
los legisladores y decisores a que destinen recursos al diseño e implementación de estrategias para evitar o, como mínimo, neutralizar estos conflictos.

Sin quererle quitar importancia al libro y reconociendo que hay medicamentos que son esenciales para la vida, me gustaría hacer una precisión. Al principio del libro, el doctor Barros atribuye los avances en los indicadores de salud al acceso a los medicamentos. Como especialista en salud pública, debo enfatizar que los factores socioeconómicos, la higiene, la educación y lo que ahora se reconoce como sociodeterminantes de la salud han tenido mayor impacto en la salud que los medicamentos. En realidad y debido a los factores que tan elocuentemente se presentan en este libro, los medicamentos son armas de doble filo. Bien utilizados salvan vidas, pero cuando se prescriben en base a información engañosa pueden provocar efectos adversos e interacciones que pueden llegar a requerir la hospitalización del paciente e incluso pueden ocasionar la muerte. Solo en Estados Unidos, el país donde más se ha estudiado este tipo de problemas, las reacciones adversas a medicamentos ocasionan más de setecientas mil consultas de emergencia y más de cien mil hospitalizaciones y muertes anuales.

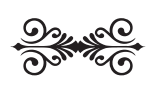

